\title{
Characterisation of Unstable Blinking Pixels in the AisaOWL Thermal Hyperspectral Imager
}

\author{
Laura Harris, Gary Llewellyn, Hannu Holma, Mark A. Warren and Daniel Clewley
}

\begin{abstract}
The AisaOWL thermal hyperspectral instrument, manufactured by Specim, is a relatively new push-broom sensor well suited to airborne environmental surveys. The sensor covers the 7.6 to 12.6 $\mathrm{\mu m}$ part of the Long Wave Infra-red region with 102 continuous bands, and is capable of imaging in low-light conditions. The detector array is a Mercury Cadmium Telluride (MCT) semiconductor, which has an inherent randomly varying dark current for random pixels. This manifests in the raw data as a pixel switching between different intensity levels. These pixels are termed "blinkers" by the manufacturer. For each data acquisition, the pixels need to be tested for blinking behaviour as different pixels are affected during each acquisition. However, little is known about the number of blink events, the duration of frames or the optimal length of data acquisition. This study presents a characterisation of the blinking nature of pixels in the MCT detector array to provide guidance in data acquisition and processing. The work finds that blinking behaviour is not completely random, with some pixels more prone to blinking behaviour than others. Most blinking pixels have only a few short blinks, therefore there is still a considerable amount of good data in a blinking pixel.
\end{abstract}

Index Terms-AisaOwl, calibration, hyperspectral, thermal.

\section{INTRODUCTION}

$\mathbf{T}$ HERMAL remote sensing is an established technique for measuring thermally emitted radiation over large, potentially hazardous regions. This information can then be used to derive temperature and emissivity [1]-[3]. Typical applications of thermal remote sensing include material classification (particularly minerals) [4], fire detection [5], assessing vegetation health [6], [7], improving energy efficiency [8], monitoring pollution emissions [9] and examining the urban heat island phenomenon [10].

Whilst there are other airborne thermal sensors available, such as the Telops HYPER-CAM and the Itres Thermal Airborne Spectrographic Imager (TASI), the AisaOwl has been selected by the Natural Environment Research Council (NERC) to provide an airborne environmental data service. This service is delivered by the NERC Airborne Research

This work was supported by the Natural Environment Research Council under the NERC Airborne Research Facility.

L. Harris, M. A. Warren, and D. Clewley are with Plymouth Marine Laboratory, Prospect Place, Plymouth, UK (e-mail: lah@pml.ac.uk; mark1@pml.ac.uk; dac@pml.ac.uk).

G. Llewellyn is with British Antarctic Survey, High Cross Madingley Road, Cambridge, UK (e-mail: gaew@nerc.ac.uk).

H. Holma is with Specim, Spectral Imaging Ltd., Oulu, Finland, (e-mail: hannu.holma@specim.fi).
Facility (ARF), which consists of the operational facility located at British Antarctic Survey and the Data Analysis Node (DAN) hosted at Plymouth Marine Laboratory. Thus characterisation of this sensor has the potential to benefit a number of researchers, each with individual applications as diverse as Agriculture-Health-SPECTrometry (AHSPECT) and Environmental Mapping and Monitoring of Iceland by Remote Sensing (EMMIRS) funded by the European Facility for Airborne Research (EUFAR) FP7.

The AisaOWL thermal hyperspectral instrument, manufactured by Specim, is a relatively new push-broom sensor ideal for airborne environmental surveys. It is sensitive to Long-Wave Infra-Red (LWIR) radiation, specifically 7.6 to $12.6 \mu \mathrm{m}$. As an airborne sensor it offers a solution to rapid demand and high resolution data. It also has the potential to compliment and calibrate data acquired from a satellite system [11], for example the Hyperspectral Thermal Emission Spectrometer (HyTES) [12], which when operated from a satellite provides coverage over larger areas without any airspace restrictions. An AisaOwl sensor has already been used to improve mineral classification of the Makhtesh Ramon in Israel [13], [14]. Table I provides an overview of the sensor specification; further details are available from the manufacturer (http://www.specim.fi/products/aisaowl/).

TABLE I: Specification of the AisaOwl

\begin{tabular}{|c|c|}
\hline Measurement & Value \\
\hline Spectral bands & 102 \\
Spatial pixels & 384 \\
Measured FOV (total) & $24.20^{\circ}$ \\
Measured FOV (from centre) & $-11.79^{\circ}, 12.41^{\circ}$ \\
Paraxial FOV & $-11.80^{\circ} 12.39^{\circ}$ \\
Sensor IFOV & $0.063^{\circ}$ or $1.103 \mathrm{mrad}$ \\
Lens focal length & 42.886 \\
Optical axes position (central pixel) & 187.7 \\
Max distortion & $0.0755 \mathrm{~mm}$ or $0.0210^{\circ}$ or $0.171 \%$ \\
\hline
\end{tabular}

Intrinsic to the AisaOwl is the Mercury Cadmium Telluride (MCT) detector array, which has an inherent randomly varying dark current for a small changing proportion of pixels. This manifests in the raw data as a pixel switching between different intensity levels. These pixels are termed "blinkers" by the manufacturer. They react as expected to different light intensities, apart from this switching behaviour, so are not bad pixels (i.e. wholly defective). Blinking behaviour occurs randomly, so pixels must be identified for each data acquisition. Pixels 
are identified by spatial position (sample), band number and acquisition time (frame number).

This paper presents a characterisation of these blinkers to provide an indication of the effect these have on sensor performance, and to determine whether data acquisition or processing can be optimised to improve the quality of the calibrated data. Information on the frequency and duration of the blinks may then be used to inform the development of a blinker detection and correction method for image data, which will need to cope with various scene features, but this is outside the scope of this work.

In order to fully characterise the blinker behaviour it was necessary to develop a new blinker detection method. This provides total visibility of all the methods used as well as the ability to use all the information, rather than just the dark-frames as Specim's tool does. Therefore blinkers can be examined for much longer timescales. This may be incorporated into a blinker detection routine for image data in the future by providing high confidence decisions about the state of each pixel just after acquisition, but on its own it cannot identify blinks within the image data. This will be essential to provide a per-pixel quality mask and attempt any correction of the blink data.

The paper is organised as follows. Section II provides an overview of the methodology used and a description of the two blinker detection routines. Section III presents the results of the tests, which are further discussed in section IV. Finally, conclusions about the AisaOWL blinkers are summarised in section V.

\section{Methodology}

The end-of-season maintenance period provides an opportunity to perform a bench calibration and characterisation of each of the sensors. The AisaOwl is calibrated using internal black bodies for each data acquisition so does not undergo the same bench calibration as the VNIR-SWIR hyperspectral sensor operated concurrently. However, this does provide an opportunity to characterise the AisaOwl using controlled temperature black bodies, which provide a spectral output that can be predicted with Planck's Law [15] for Black Body radiation:

$$
B_{\lambda}(T)=\frac{2 h c^{2}}{\lambda^{5}} \times \frac{1}{e^{h c / \lambda k_{B} T}-1}
$$

where $B_{\lambda}(T)$ is the radiant energy at wavelength $\lambda$ and temperature $T, h$ is Planck's constant, $c$ is the speed of light and $k_{B}$ is the Boltzmann constant.

Data from two calibration events have been used in this study, 2014 and 2016. During the 2014 calibration event data from 25 separate acquisitions were collected. These acquisitions varied in integration time and temperature, but had durations of only a couple of minutes. Blinkers from this calibration event are characterised using Specim's preprocessing tool, which only tests the dark-frames (i.e. data collected with a closed shutter), so actual data acquisition duration is irrelevant. The numbers of blinkers for each band and sample have been examined in this data to identify any trends.

During the 2016 calibration event, data were collected specifically to characterise detector stability and blinking pixels, so included longer acquisitions than normal. Over the course of two days 4 long acquisitions of approximately 3 hours were recorded, with dark-frames and internal black body data captured approximately every 10 minutes, but with 3 longer uninterrupted acquisitions lasting 47, 64 and 89 minutes. For all experiments the frame rate was set to 100 frames per second (fps), so a dark-frame acquisition of 1024 frames corresponds to just over 10 seconds of data. These data are processed with Specim's preprocessing routine and also with the in-house developed method to examine all of the data, rather than just the dark-frames.

Trends in blinker frequency for band and sample are examined in the 2016 data using the developed blinker detection routine, as well as the number and duration of individual blinks. The impact of file duration is also examined to identify whether there is any benefit in altering the duration of data acquisition. Finally, the overall results between the two detection methods are compared.

\section{A. Specim Blinker Detection Routine}

Specim provide a preprocessing tool to radiometrically calibrate the raw data, which also tests for blinking pixels. A pixel map is produced of all of the blinkers consisting of a binary matrix the size of the detector array. Along with the calibrated data and the calibration data derived from the black body data, this pixel map is a saved output of the tool, so can be used to mask the blinking pixels in the calibrated data if required. However, the tool also removes all blinking pixels from the processed data and replaces them with good data from a nearby pixel, regardless of whether the blinking pixel may have good data. Thus blinking pixels cannot be examined in the processed data. In fact, only the dark-frames are tested to identify blinking behaviour. For this reason, Specim recommend a long dark-frame capture (at least 1024 frames) to improve the identification of blinking pixels.

All of the data were processed using version 2.6.3 of Specim's tool with the default settings using the blinker detection threshold of 0.011. Upon modification of this threshold the blinker detection results could be changed, but an unacceptable loss in data was observed so an alternative method for blinker detection and treatment was initiated.

\section{B. NERC-ARF Blinker Detection Routine}

In order to fully characterise the blinker behaviour it was necessary to develop a new blinker detection method. The 
developed routine allows total visibility of all the procedures implemented and can be used directly on the raw data itself to examine blinkers for much longer timescales, unlike Specim's preprocessing tool which only performs blinker detection on calibrated dark-frames. Furthermore, this allows only the blinking data to be masked, as is the preferred NERC-ARF method, rather than the whole time series replaced. This detection algorithm may be directly applied to the black body calibration data to eliminate the effect of blinking data in the calibration procedure. It may not be used directly on the image data as it assumes a constant signal level throughout the time series.

As previously noted a blinking pixel is defined as a pixel that changes output signal between two different levels. Thus the blinker detection routine has been designed to examine each pixel frame series for two distinct output levels by converting the frame series into an histogram. The raw data is used here rather than the calibrated data. This means that the blinker detection should not be affected by problems in the calibration which may arise due to blinkers or pixel overflows in the internal black body data. The blinker detection routine is illustrated as a flow diagram in Fig. 1.

Pixels with more than two output levels are not considered blinkers for this characterisation as it is more difficult to determine which output level is correct and it becomes extremely difficult to distinguish between noisy pixels and blinking pixels. Furthermore this prevents the derivation of statistics on the number and duration of blinks i.e. the frames in which a blinking pixel has an incorrect output.

Any pixel with an output signal that significantly drifts throughout data collection cannot be examined for blinking behaviour using a histogram approach. This is because the blinks appear at different output intensities and the non-blink frames obscure any peaks in the histogram distribution. Thus pixels are first tested for drift from their gradient and ignored as bad pixels if a threshold of 0.25 is exceeded.

In order to reject bad pixels that are also blinking and some noisy pixels that may appear to be blinking in histogram space, particularly for short data acquisitions, some analysis of the histogram peaks is conducted. Peaks too close together, too wide and too low in intensity relative to neighbouring minima are ignored. It is advantageous to distinguish between bad and blinking pixels, as data from bad pixels are always unreliable whereas data from blinking pixels can be used depending on the state.

Once a pixel has been identified as a blinker, the number and duration of blinks are calculated. The blinking level is identified as the least populated of the two, limiting the blink duration measurement to half the file length. The variation allowed by the blinking state is determined from the width of the blink histogram peak. If there is not much separation between the output levels of the blinker, some noisy frames may be counted as blinks. These typically only last for a single

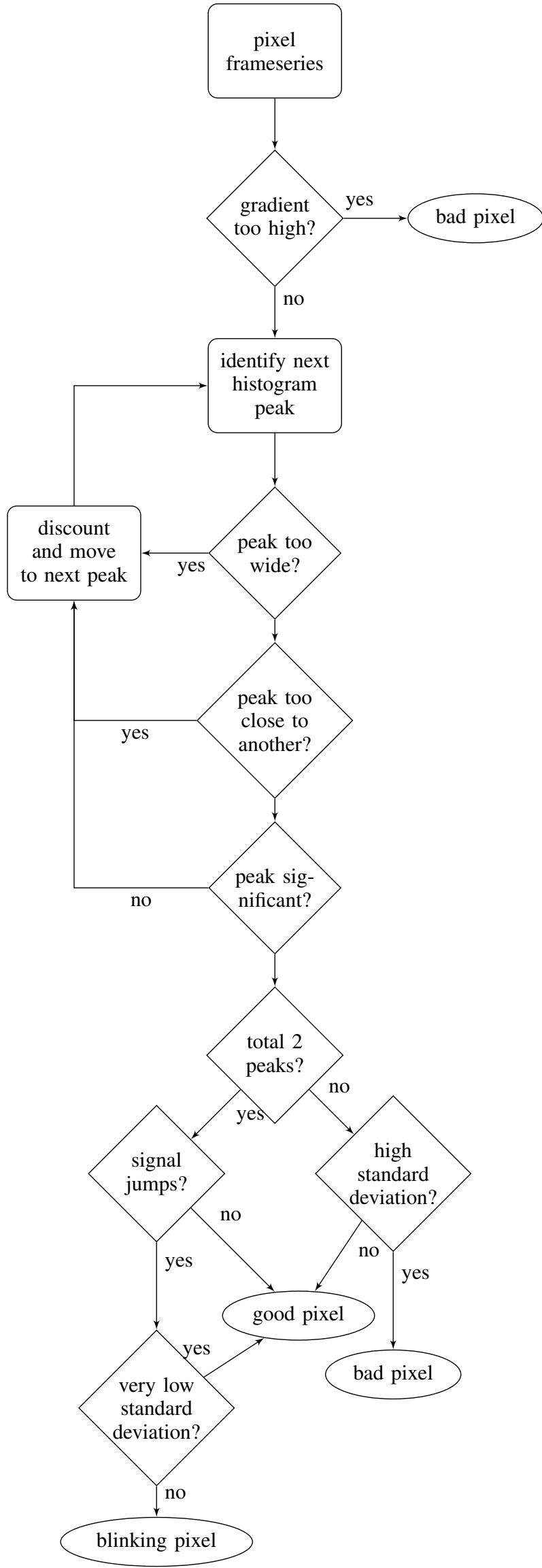

Fig. 1: Flow chart representation of the NERC-ARF blinker detection routine. 
frame so can be ignored. However, some well defined blinks do last a single frame, so ignoring them would also skew the blinker statistics. For this analysis, single frame blinks / noise have been included.

Some examples of the different pixel behaviour are illustrated in Fig. 2, highlighting the difficulties in setting the boundaries between the different pixel types. The standard deviation threshold of 10 between good and bad pixels lies between pixels a and $\mathrm{c}$, where a is labelled good and $\mathrm{c}$ bad. Pixel e highlights the problem with noisy blinkers which do not have much of a gap between the different blinking states. Some of the noise is counted as a blink, skewing the results for the number and duration of blinks. Pixel $b$ is identified as a frequently blinking pixel because the range of variation is larger than the acceptable threshold, which has been determined from manual comparison of a large number of pixels to give high quality data. Noisy pixels are more irregular in their variation, whereas this pixel is alternating between 2 different states.

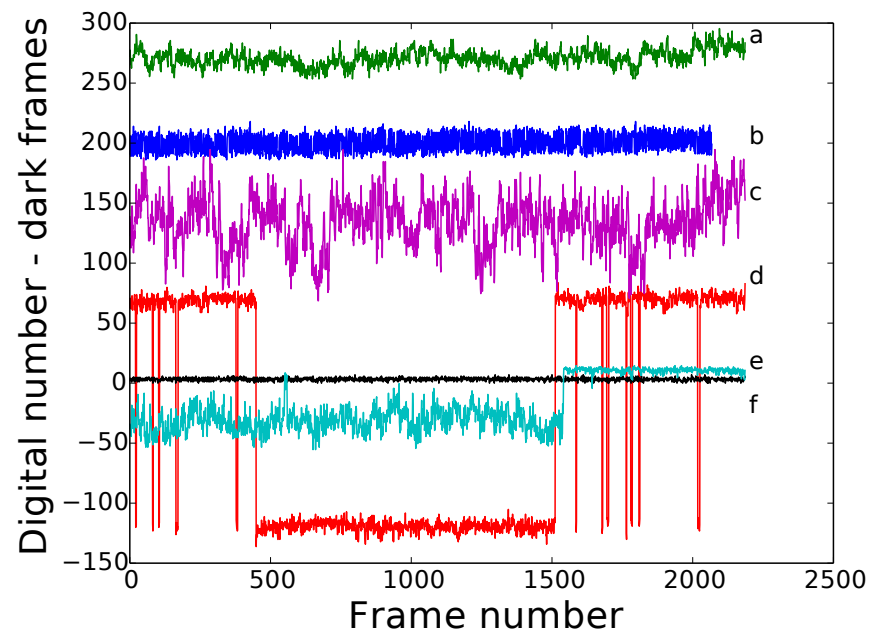

Fig. 2: Example pixel frame series of approximately 22 seconds plotted as raw digital number with the average of the dark-frames subtracted, except for (a) and (b), which have been vertically offset by +50 for clarity. a: good pixel with high standard deviation, b: frequently blinking pixel, c: bad pixel, d: pixel with strong blinks, e: noisy blinking pixel, f: good pixel.

It is clear that bad pixels have a greater standard deviation than good pixels. Blinking pixels have an average standard deviation in between good and bad pixels, but with a greater range. Tables II - IV show the statistics of the standard deviations for three different acquisitions at three different temperatures. Because of the overlap between the distributions it is impossible to characterise a pixel as a blinker just by standard deviation alone. The data also show that temperature has little impact.
TABLE II: Statistics of $40^{\circ}$ data

\begin{tabular}{|c|c|c|c|}
\hline & Good & Bad & Blinking \\
\hline Number of pixels & 36180 & 2602 & 386 \\
Mean of stdev & 3.42 & 21.18 & 13.52 \\
Stdev of stdev & 1.46 & 15.27 & 21.65 \\
\hline
\end{tabular}

TABLE III: Statistics of $60^{\circ}$ data

\begin{tabular}{|c|c|c|c|}
\hline & Good & Bad & Blinking \\
\hline Number of pixels & 36387 & 2386 & 395 \\
Mean of stdev & 3.38 & 20.93 & 13.20 \\
Stdev of stdev & 1.43 & 15.82 & 21.24 \\
\hline
\end{tabular}

TABLE IV: Statistics of $80^{\circ}$ data

\begin{tabular}{|c|c|c|c|}
\hline & Good & Bad & Blinking \\
\hline Number of pixels & 36429 & 2393 & 346 \\
Mean of stdev & 3.41 & 20.47 & 14.63 \\
Stdev of stdev & 1.43 & 13.66 & 24.84 \\
\hline
\end{tabular}

\section{RESULTS}

\section{A. Initial Study with the Specim tool}

Blinking pixels were compared from 25 data sets acquired in 2014 to determine which pixels, if any, were more prone to blinking than others. Only the dark-frames of each data set are used. Various integration times and temperatures have been used for each of the dark-frame acquisitions of 1024 frames. The average number of blinking pixels per band across all of the data sets is plotted in Fig. 3, clearly showing a much greater chance of a blinking pixel for the higher bands (band 80 - 102) compared to lower bands, particularly bands $40-60$. This effect is partially due to the reduced sensitivity of these bands, which require higher calibration multipliers and therefore deviation in the time series is more apparent to Specim's tool. Further investigation has identified that some of the higher bands may be approaching saturation in the high temperature internal black body data, which corrupts the calibration factor and the calibrated data. This may also have an impact on blinker detection in the highest bands. Fig. 4 shows that the pixel spatial position does not have a significant effect on blinking behaviour.

The total number of times that each pixel was recorded as a blinker for all 25 data sets is plotted in Fig. 5. This indicates that there are a large number of pixels that are only blinkers 3 or 4 times within the 25 data collections. In fact, $35.6 \%$ of the data fall within these bins. There are $30.1 \%$ of pixels that are blinkers more than 10 times, and $9.1 \%$ more than 20 times. Some pixels do not blink at all for any of the 25 data acquisitions. There are also some that blink consistently in every data collection. This suggests that some pixels are more prone to blinking than others. 




Fig. 3: Average number of blinking pixels per band for 25 different data sets.

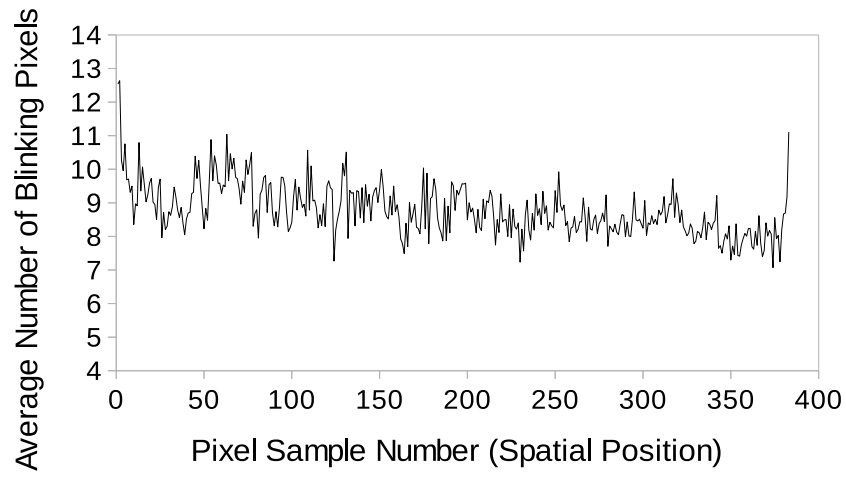

Fig. 4: Average number of blinking pixels per pixel sample number (i.e. spatial position across the detector array) for 25 different data sets.

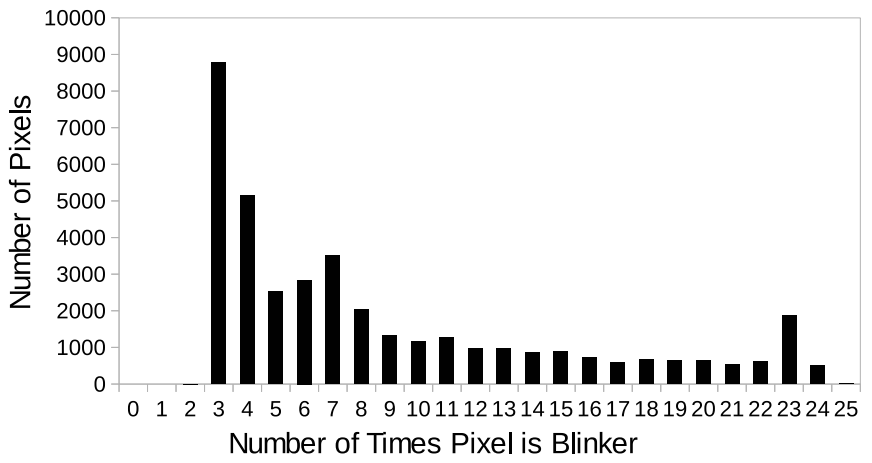

Fig. 5: Total number of times that each pixel band and sample was recorded as a blinker for all 25 data sets.
Before applying the developed blinker detection routine to the test data the routine was manually validated with 2 methods. One thousand randomly selected pixels were inspected and manually scored as either good or bad/blinking. These were then compared to the results achieved with the NERC-ARF blinker detection routine. Only 1 pixel was mislabelled a blinker and only 2 blinkers were missed.

The second method for validating the results uses the processed image. Blinking and bad pixels are very visible in the processed data, so comparing processed data of a uniform black body target with and without the identified bad and blinking pixels masked out provides an efficient method of validation. Fig. 6a shows some an example of part of the image data, which is reproduced in Fig. 6b with just the blinkers masked and again in Fig. 6c with just the bad pixels masked. The bad pixels are masked for the whole time series so show as a vertical black bar in the masked image whereas the blinking pixels are only black in the masked image where a blink has been detected. Because of the contrast in the original image it is quite difficult to see all of the the bad and blinking pixels at once, but the blinking pixel becomes more obvious once the bad pixels are masked out. Three bad pixels are detected in this example and one blinker, spatially adjacent to the third bad pixel.

Results of this comparison show that most blinkers are successfully identified in the data, but some bad pixels need to be identified from comparison with neighbouring pixels when there are no anomalies in the dark-frame frame series. This occurs when a bad pixel has no response or when the output is continuously at the wrong level, possibly because the pixel has blinked between acquisition of the internal black body data and image data, inferring that the calibration data for that pixel is no longer appropriate. Some pixels were masked that did not appear as bright stripes in the image, particularly low standard deviation blinkers. Perfect identification of every good, bad and blinking pixel for every data set is not expected due to the imprecise definition of each of these pixel states.

The NERC-ARF blinker detection routine has been applied to all of the data specifically collected for blinker characterisation during the 2016 calibration event. The number of bad pixels averaged by band and sample for all of the 4 long acquisition sets are plotted in Fig. 7 and Fig. 8. A trend is clearly visible across the bands, oscillating around 3.5 bad pixels for the first 60 bands and then rapidly decreasing until there are almost no bad pixels by band 100 . There is also an observable difference between the 2 sides of the detector. For the first 200 samples (i.e. spatial positions) the average number of bad pixels is generally higher than samples $200-384$.

Plotting similar graphs in Fig. 9 and Fig. 10 for the number of blinking pixels shows different trends. Blinking pixels are more likely in bands $25-93$ and samples $100-170$, though there is a lot of deviation between adjacent samples. 


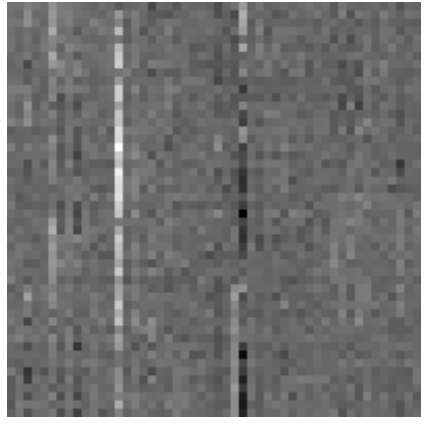

(a) No mask applied.

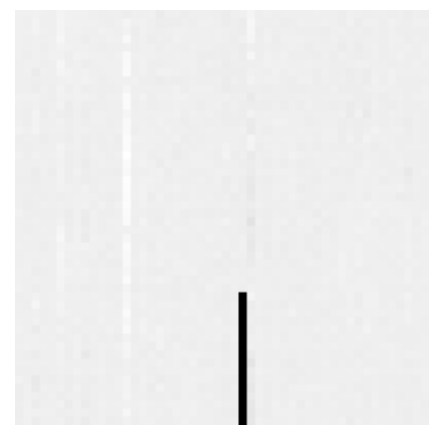

(b) Detected blinking pixels masked.

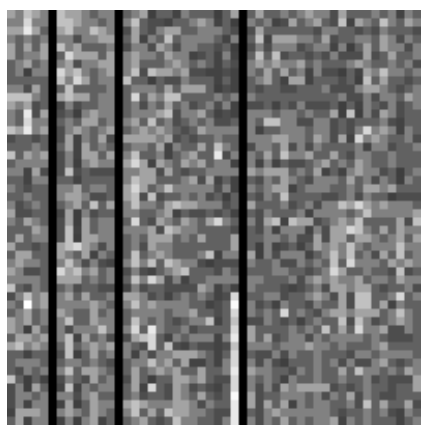

(c) Detected bad pixels masked.

Fig. 6: Calibrated dark-frames showing the bad and blinking pixels of a single band before and after masking.

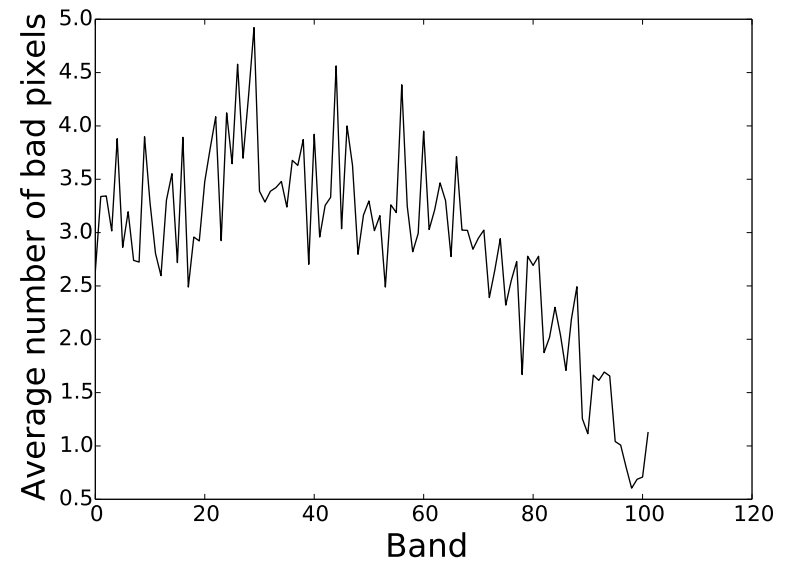

Fig. 7: Average number of bad pixels detected for each band.

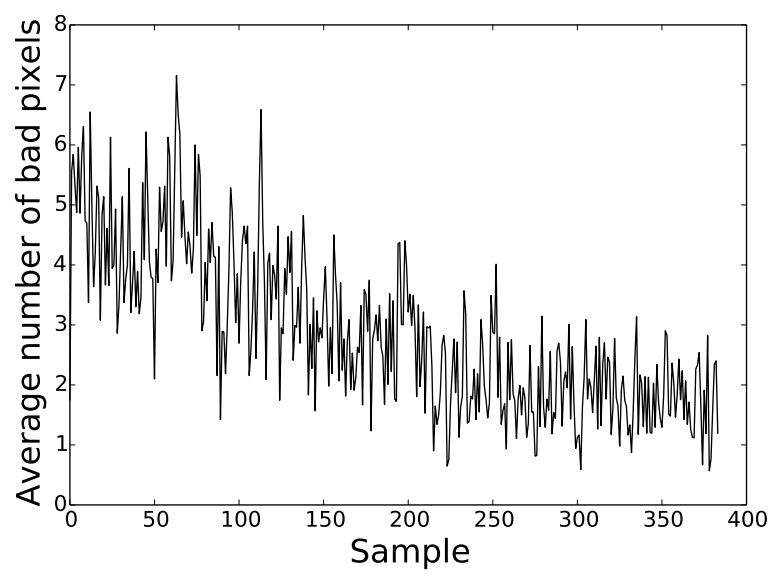

Fig. 8: Average number of bad pixels detected for each sample.

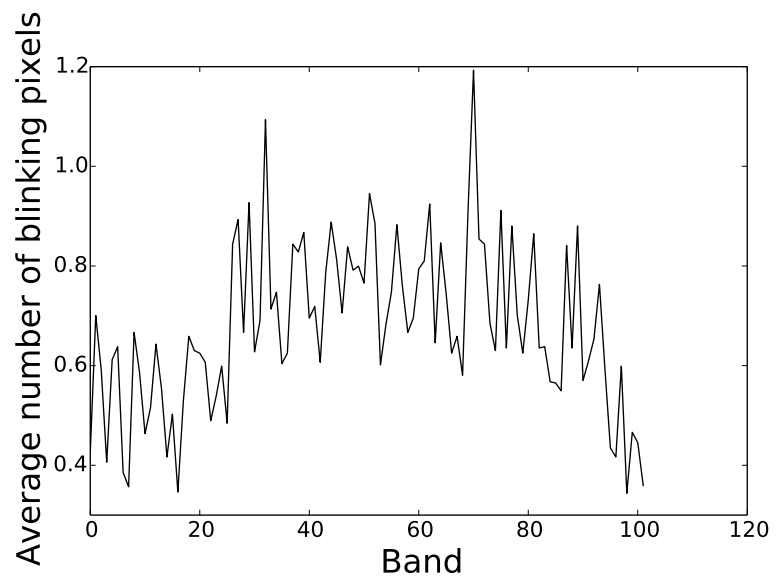

Fig. 9: Average number of blinking pixels detected for each band.

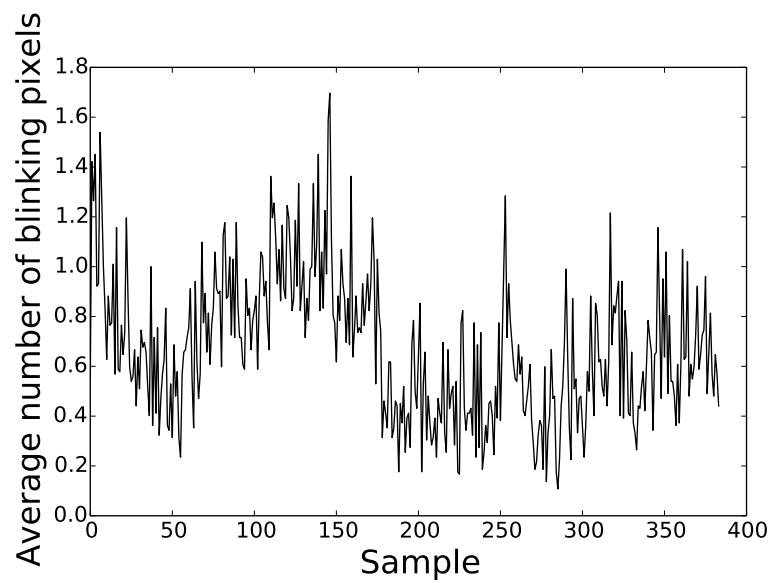

Fig. 10: Average number of blinking pixels detected for each sample. 


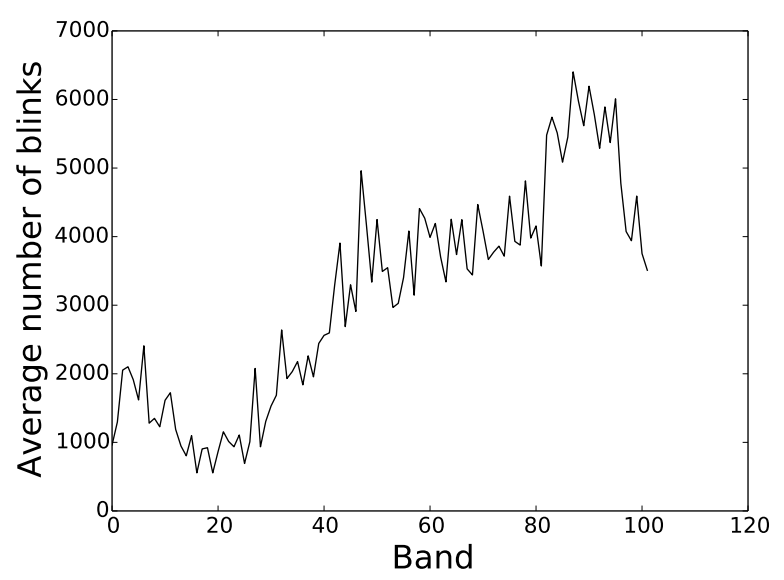

Fig. 11: Average number of blinks detected for each band. Note that no trend is observed for all data files except for the longest 89 minute acquisition.

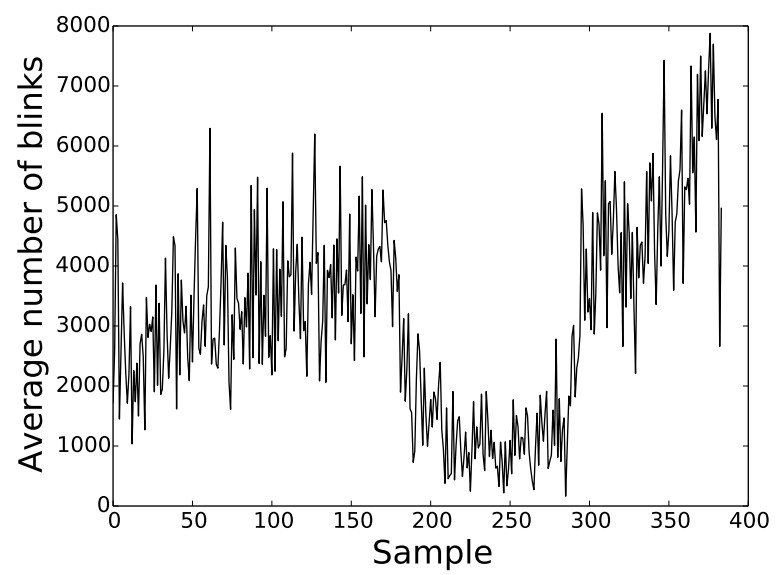

Fig. 12: Average number of blinks detected for each sample. Note that no trend is observed for all data files except for the longest 89 minute acquisition.

Examining the number of blinks for each pixel across all the files shows no trend with band or sample, except for the 89 minute file. The number of blinks for all files are shown in Fig. 11 and Fig. 12, where the very large number of blinks in the 89 minute file dominates the results. The number of blinks is much greater in the higher bands, suggesting that these bands have become more unstable during the longest acquisition. Samples 190 - 290 appear more stable as they have fewer blinks.

As well as the differences in the affinity of some bands towards blinking behaviour, the developed blinker detection routine generally identifies more pixels as normal rather than blinking or bad. Table $\mathrm{V}$ shows the percentages of pixels that remain good or bad for an entire long acquisition. Note that there are differences between the two methods, as Specim's method does not distinguish between bad and blinking pixels. Therefore, the pixels not included in the Specim columns are
TABLE V: Good and Bad Pixel Percentages for the Four Long Acquisition Sets Calculated Using Specim's Tool and the NERC-ARF Method.

\begin{tabular}{|c|l|l|l|c|}
\hline \multirow{2}{*}{$\begin{array}{c}\text { Long } \\
\text { Acquisition }\end{array}$} & \multicolumn{2}{|c|}{ Specim } & \multicolumn{2}{c|}{ NERC-ARF } \\
\cline { 2 - 5 } & $\begin{array}{l}\text { Always } \\
\text { Good }\end{array}$ & $\begin{array}{l}\text { Always } \\
\text { Bad }\end{array}$ & $\begin{array}{l}\text { Always } \\
\text { Good }\end{array}$ & $\begin{array}{l}\text { Always } \\
\text { Bad }\end{array}$ \\
\hline 1 & N/A & N/A & $95.26 \%$ & $0.51 \%$ \\
2 & $74.88 \%$ & $9.39 \%$ & $92.65 \%$ & $0.32 \%$ \\
3 & $81.62 \%$ & $14.44 \%$ & $68.68 \%$ & $6.21 \%$ \\
4 & $81.61 \%$ & $14.48 \%$ & $92.02 \%$ & $5.90 \%$ \\
\hline
\end{tabular}

switching between good and bad for different data acquisitions, but pixels not included in the NERC-ARF columns may also be blinking. This is a major cause of the lower percentage of pixels found as bad for every acquisition by the NERC-ARF routine. However, good data exists in blinking pixels, so it is important to make the distinction.

There are no Specim results for long acquisition set 1 as the sensor failed to collect calibration data and so data could not be processed with Specim's tool. The other results suggest that the default threshold for Specim's tool is too high and it should be tuned according to each data set. The number of blinking pixels observed in the processed data and the time that it would take to iteratively tune the blinking pixel threshold for each data file is strong justification for the implementation of the developed blinker detection routine into the NERC-ARF automated processing chain. The reason for the large increase in blinking pixels in long acquisition set 3 is unknown, but may just be because this included the 89 minute acquisition, so more pixels have started to blink within the acquisition time.

The number of blinks and duration of each blink have been examined in these data. Both of these measures depend on the file length, so have been examined for all files as well as similar length files. The frequency of the various numbers of blinks is plotted in Fig. 13 for the 89 minute file as a percentage distribution. Of all the pixels, $87 \%$ blink more than 5 times throughout this acquisition, but $10 \%$ of pixels blink more than 99128 times. The fall off in blinking number is exponential, but shows a linear distribution when plotted on a cumulative scale. It can be approximated by the dot-dashed line with slope -0.00079 , intercept $88, R^{2} 0.96$ and standard error $4.0 \times 10^{-5}$. For all other files the fall off in number of blinks is exponential on the cumulative scale, indicating that the longer duration of this data acquisition has had an adverse effect on pixel stability (and therefore data quality) because a higher percentage of pixels have more blinks. This file was acquired after other data acquisitions, so the sensor had actually been acquiring data for 3.5 hours before starting this acquisition, which may also have impacted the stability.

Fig. 14 shows the frequency of the durations of each blink within the 89 minute data set only, to avoid the large number of short files distorting the results. The distribution is similar for all files, showing a very large amount of blinks with short 


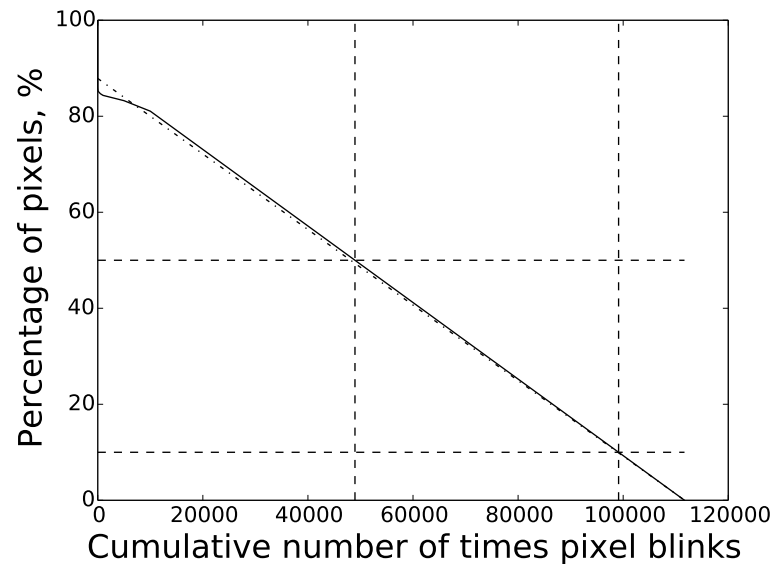

Fig. 13: Percentage of the blinking pixels blinking greater than each number. Of the blinking pixels, $86 \%$ blink more than once, $50 \%$ more than 27 times and $10 \%$ more than 4333 times. The distribution can be represented by the dot-dashed line with slope -0.00079 , intercept $88, R^{2} 0.96$ and standard error $4.0 \times 10^{-5}$.

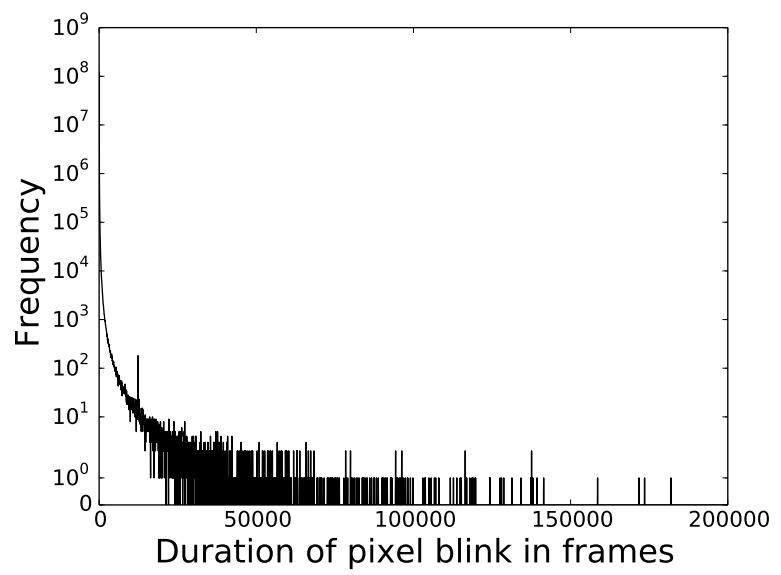

Fig. 14: Frequency of blink durations for the longest data acquisition.

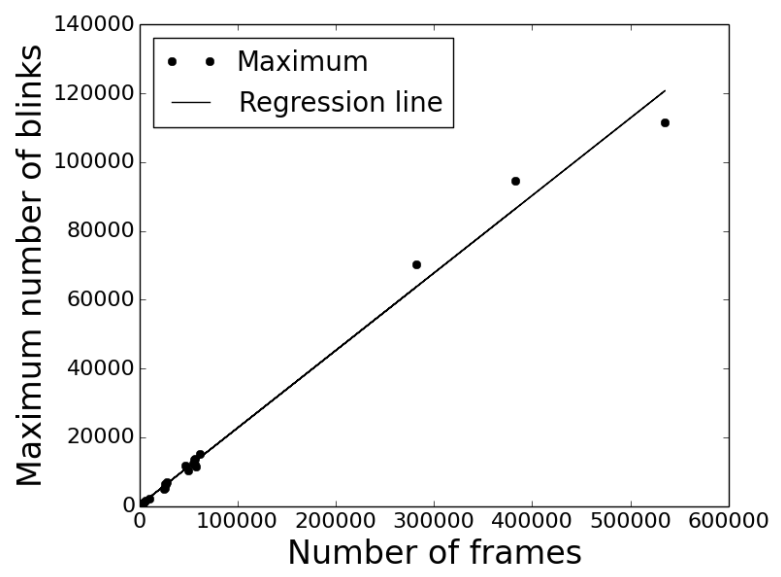

Fig. 15: Maximum number of blinks for each file of different frame length. durations and much fewer blinks with longer durations. The blink duration for this file falls off rapidly on an exponential scale with a maximum duration of 209407 frames. Only three blinks have a duration greater than 200000 , outside the range plotted.

Both the total blink duration and the total number of blinks increase linearly with file length $\left(R^{2}=0.99\right)$, indicating that the overall probability of blinking pixels occurring remains constant over the length of the file. Analysing the number of blinks and their duration for each of the similar length files does not show any significant trends with time from switch on. Fig. 15 shows the maximum number of blinks for each file against the number of frames in the file.

\section{DISCUSSION}

Examining the maximum duration of a blink against the maximum permitted by the method and length of file provides an indication of how the results are affected by the methodology. Although the methodology does pose a maximum limit on the blinker duration, in reality this is less of a problem because any blink that lasts the entire duration of a data acquisition including calibration data acquisition from the black bodies is actually a good pixel for that file. For pixels with long blink durations it is beneficial to acquire internal black body data regularly so that they can be correctly calibrated in the raw data with no blinking problems. Given that most blinks are very short in duration, shorter data acquisitions are not expected to decrease the number of blinks considerably. Hence, there is very little benefit in altering file length to reduce the effect of blinkers. However, the increase in the number of blinks for the longest acquisition of 89 minutes suggests that the detector may be becoming more unstable, so acquisition length may eventually affect data quality. This file was acquired after other data acquisitions, so the sensor had actually been acquiring data for 3.5 hours before starting this acquisition, which may also have impacted the stability.

Previous characterisation of the AisaOwl identified reduced sensitivity in the higher bands [16], which could result in less variation in the frame series (i.e. variation in a pixel output with time) and hence fewer bad pixels. These bands appear to have become more unstable for the longest data acquisition of 89 minutes as a higher number of blinks are observed in the higher bands. However, even the lower bands have ten times the number of blinks that would be expected of a file of this frame duration given the trend observed from all the other files. The slight bias of bad pixels on one side of the detector array cannot be explained, but may be a result of the manufacturing process.

Manual checking of pixel frame series does provide confidence in the blinker detection results, including the number and duration of blinks, but it is impossible to check each of the 39167 pixels for every data file. One thousand randomly selected pixels have been manually scored as either good or 
bad/blinking and compared to the results with both the NERCARF developed routine and Specim's tool at different bad pixel thresholds. The resulting Receiver Operator Characteristic curve for the different thresholds is shown in Fig. 16 along with the NERC-ARF detection results. The default value for the Specim tool detects almost all of the bad or blinking pixels, but labels many good pixels as blinkers. Increasing the threshold does reduce the number of false alarms, but at the expense of blinker detection. The NERC-ARF routine achieves better results with almost no mislabelled good pixels and only two missed bad pixels.

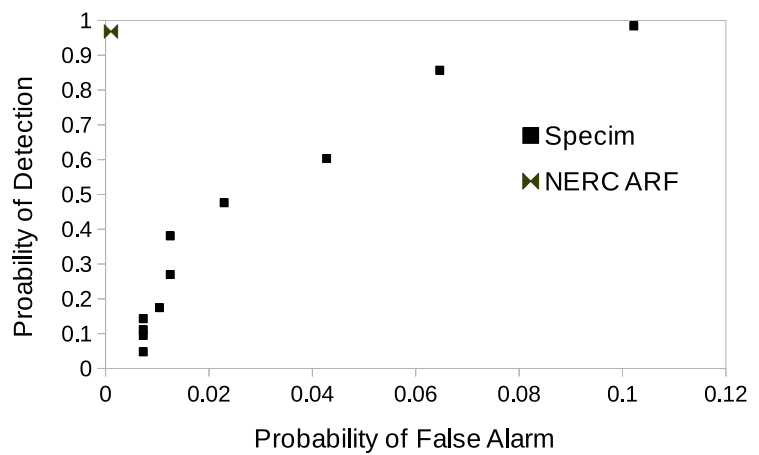

Fig. 16: Receiver Operating Characteristic curve of the results obtained with Specim's tool with varying blinker detection threshold on 1000 random pixels. The results for the NERCARF detection routine are also shown.

An anomalous result was observed for one data acquisition, which has been excluded from the statistical analysis. For this data acquisition $21 \%$ of the pixels were found bad and $73 \%$ blinking with the NERC-ARF detection routine. Most of this increase in bad pixels is observed in the fist 50 bands, whereas the increase in blinkers is more evenly distributed if the effect of the bad pixel distribution is ignored. For this data acquisition, a sudden random jump in output level for every pixel is observed in the raw data. The cause of this detector jump is unknown, but means that the same calibration cannot be applied to data before and after then jump.

\section{CONCLUSION}

Data from an AisaOwl thermal hyperspectral instrument have been analysed to determine the stability of the detector with regards to blinking pixels. Two methods have been compared: the supplied manufacturer method (Specim v2.6.3) and an inhouse (NERC-ARF) developed algorithm. Both methods show that the behaviour of blinking pixels is not entirely random, but they also both identify different pixels as the most likely blinkers. Correct identification of blinking pixels is extremely important because a blinking pixel does have good data at some times and these need to be kept.

The manufacturer method uses calibrated dark-frames to detect the blinking pixels whereas the NERC-ARF method uses the complete raw data acquisition. This likely means that the manufacturer method is impacted by band sensitivity and uncertainties in the black body data. By using the raw data the NERC-ARF method has the advantage that these uncertainties in the black body data (such as overflows and blinking pixels) have no impact. The Specim method has been improved upon since v2.6.3.

The Specim method blinking pixel detection results are controlled by a threshold, but changing this either increases the number of false detections or decreases the number of detected blinkers, as shown in Fig. 14. The NERC-ARF method produced better results when compared against manual verification.

No trends were found in the number of blinks during data acquisition for all but one acquisition, with a duration of 89 minutes. This had increased blinks in the higher bands suggesting this region of the detector had become unstable. This file was acquired after other data acquisitions, so the sensor had actually been acquiring data for 3.5 hours before starting this acquisition. Therefore it is recommended that long data acquisitions are avoided. Aside from this, blinker frequency and duration are independent of acquisition duration, so the data acquisition procedure cannot be optimised to improve the data quality.

It was found that most pixels only blink for a short duration. Thus it may be possible to recover data from blinking pixels without needing any correction. However an accurate method for identifying the blinks in real image data needs to be developed to achieve this. This should be targeted towards detecting short duration blinkers as these are the most common, but there are no other rules on blinker duration and frequency. The developed method of blinker detection may already be used on the black body calibration flight data to eliminate the effect of blinking data in the calibration procedure.

\section{ACKNOWLEDGEMENT}

The authors would like to thank Chris MacLellan of the NERC Field Spectroscopy facility for his assistance in instrument calibration.

All data used in this study have been provided by the Natural Environment Research Council (NERC) Airborne Research Facility.

\section{REFERENCES}

[1] A. Gillespie, S. Rokugawa, T. Matsunaga, J. D. Cothern, S. Hook, and A. B. Kahle, "A temperature and emissivity separation algorithm for Advanced Spaceborne Thermal Emission and Reflection Radiometer (ASTER) images," IEEE Transactions on Geoscience and Remote Sensing, vol. 36, pp. 1113-1126, 1998. DoI: http://dx. doi.org/10.1109/36.700995. 
[2] J. A. Morgan, "The definition of surface emissivity in thermal remote sensing," Aerospace Conference, 1998 IEEE, vol. 5, pp. 159-169, 1998. DOI: http://dx.doi. org/10.1109/AERO.1998.685813.

[3] Z. Li, F. Becker, M. P. Stoll and Z. Wan, "Evaluation of Six Methods for Extracting Relative Emissivity Spectra from Thermal Infrared Images," Remote Sensing of Environment, vol. 69, pp. 197-214, 1999. DOI: http: //dx.doi.org/10.1016/S0034-4257(99)00049-8.

[4] S. Kotthaus, T. E. L. Smith, M. Wooster and C. S. B. Grimmond, "Derivation of an urban materials spectral library through emittance and reflectance spectroscopy," ISPRS Journal of Photogrammetry and Remote Sensing, vol. 94, pp. 197-212, 2014. DOI: http: //dx.doi.org/10.1016/j.isprsjprs.2014.05.005.

[5] R. S. Allison, J. M. Johnston, G. Craig and S. Jennings, "Airborne Optical and Thermal Remote Sensing for Wildfire Detection and Monitoring," Sensors, vol. 16, no. 8, 2016. DOI: http://dx.doi.org/10.3390/s16081310.

[6] J. Bellvert, P. J. Zarco-Tejada, J. Marsal, J. Girona, V. Gonzalez-Dugo and E. Fereres, "Vineyard irrigation scheduling based on airborne thermal imagery and water potential thresholds," Australian Journal of Grape and Wine Research, vol. 22, pp. 307-315, 2016. DOI: http://dx.doi.org/10.1111/ajgw.12173.

[7] J. Bellvert, J. Marsal, J. Girona, V. Gonzalez-Dugo, E. Fereres, S. L. Ustin and P. J. Zarco-Tejada, "Airborne Thermal Imagery to Detect the Seasonal Evolution of Crop Water Status in Peach, Nectarine and Saturn Peach Orchards," Remote Sensing, vol. 8, no. 1, 2016. DOI: http://dx.doi.org/10.3390/rs8010039.

[8] G. J. Hay, C. Kyle, B. Hemachandran, G. Chen, M. M. Rahman, T. S. Fung and J. L. Arvai, "Geospatial Technologies to Improve Urban Energy Efficiency," Remote Sensing, vol. 3, no. 7, pp. 1380-1405, 2011. DOI: http://dx.doi.org/10.3390/rs3071380.

[9] D. G. Streets, T. Canty, G. R. Carmichael, B. de Foy, R. R. Dickerson, B. N. Duncan, D. P. Edwards, J. A. Haynes, D. K. Henze, M. R. Houyoux, D. J. Jacob, N. A. Krotkov, L. N. Lamsal, Y. Liu, Z. Lu, R. V. Martin, G. G. Pfister, R. W. Pinder, R. J. Salawitch and K. J. Wecht, "Emissions estimation from satellite retrievals: A review of current capability," Atmospheric Environment, vol. 77, pp. 1011-1042, 2013. DOI: http: //dx.doi.org/10.1016/j.atmosenv.2013.05.051.

[10] J. A. Voogt and T. R. Oke, "Thermal remote sensing of urban climates," Remote Sensing of Environment, vol. 86, pp. 370-384, 2003. DOI: http://dx.doi.org/10.1016/ S0034-4257(03)00079-8.

[11] F. A. Kruse, J. W. Boardman and J. F. Huntington, "Comparison of airborne hyperspectral data and EO1 Hyperion for mineral mapping," IEEE Trans. Geosci. Remote Sens, vol. 41, no. 6, pp. 1388-1400, 2003. DOI: http://dx.doi.org/10.1109/TGRS.2003.812908.

[12] W. R. Johnson et al., "Hytes: thermal imaging spectrometer development," Aerospace Conference, 2011 IEEE, 2011. DOI: 10.1109/AERO.2011.5747394.
[13] G. Notesco, Y. Ogen and E. Ben-Dor, "Integration of Hyperspectral Shortwave and Longwave Infrared Remote-Sensing Data for Mineral Mapping of Makhtesh Ramon in Israel," Remote Sensing, vol. 8, no. 4, 2016. DOI: http://dx.doi.org/10.3390/rs8040318.

[14] G. Notesco, Y. Ogen and E. Ben-Dor, "Mineral Classification of Makhtesh Ramon in Israel Using Hyperspectral Longwave Infrared (LWIR) Remote-Sensing Data,' Remote Sensing, vol. 7, no. 9, pp. 12 282-12 296, 2015. DOI: http://dx.doi.org/10.3390/rs70912282.

[15] M. Planck, "Ueber das Gesetz der Energieverteilung im Normalspectrum," Ann. Phys, vol. 309, pp. 553-563, 1901. DOI: http : / / dx . doi . org / 10 . 1002 / andp . 19013090310.

[16] L. Harris, M. Warren, M. Grant and G. Llewellyn, "Spectral Characterisation of the AisaOwl," Accepted for Publication by IEEE Trans. Geosci. Remote Sens, 